equivalent to drinking two and a half times as many cups of coffee or tea each day as usual. If there is a dose response relation between coffee consumption and coronary heart disease or low birthweight babies, as seems to be the case, and that risk is mediated by caffeine then smokers who abstain would be at continuing high risk because of their caffeine consumption. Doctors offering antismoking treatment should advise patients that continued consumption of coffee at the same level may exacerbate the tobacco withdrawal syndrome and contribute to increased health risks; these patients should reduce their consumption.
This research was partly supported by grants from the National Institutes of Health.

I Istvan J, Matarazzo JD. Tobacco, alcohol, and caffeine use: a review of their interrelationships. Am Psychol Bull 1984;95:301-26.

2 Parsons WD, Neims AH. Effect of smoking on caffeine clearance. Clin Pharmacol Ther 1978;24:40-5.

3 LaCroix AZ, Mead LA, Liang K-Y, Thomas CB, Pearson TA. Coffee consumption and the incidence of coronary heart disease. $N$ Engl $f$ Med consumption and

4 Martin TR, Bracken MB. The association between low birth weight and caffeine consumption during pregnancy. Am J Epidemiol 1987;126:813-21. 5 Hall SM, McGee R, Tunstall CD, Duffy J, Benowitz NL. Changes in foo intake and activity after quitting smoking. $\mathcal{f}$ Consult Clin Psychol 1989;57 81-6.

(Accepted 25 fanuary 1989)

\section{Identifying hypoxaemia in children admitted for adenotonsillectomy}

\author{
V H van Someren, J Hibbert, J K Stothers, \\ M C Kyme, G A J Morrison
}

United Medical and Dental Schools of Guy's and St Thomas's Hospitals, Guy's Hospital, London SE1 9RT $\mathrm{V} H$ van Someren, $\mathrm{MD}$, lecturer in paediatrics J Hibbert, FRCS, consultant otorhinolaryngologist

\section{Joint Academic \\ Department of Child}

Health and Ear, Nose, and Throat Department, The London Hospital Medical College and The London Hospital, London E1 J K Stothers, $\mathrm{MB}$, senior lecturer, neonatal research group

M C Kyme, SEN, research nurse, neonatal research group G A J Morrison, FRCS, registrar

Correspondence to: Dr van Someren.

BrMed J 1989;298:1076 without recourse to studies during sleep.

\section{Subjects, methods, and results} for adenotonsillectomy.
Most children with obstructive sleep apnoea have adenotonsillar hypertrophy,,$^{1-3}$ but what proportion of the children with large tonsils and adenoids have obstructive sleep apnoea is unknown. ${ }^{45}$ We studied this and whether affected children might be identified

Forty four children (aged 2 to 7 ) were studied on the night before adenotonsillectomy. Twenty controls with no active symptoms of upper or lower respiratory tract disease were studied on the night before another elective procedure. Both groups underwent a preoperative clinical assessment by an otorhinolaryngologist, who noted symptoms, signs, and the indication

Oxygen saturation was measured continuously overnight with a pulse oximeter (Novametrix). Chest impedance was also measured and electrocardiography performed. The baseline oxygen saturation during sleep was obtained by sampling the saturation three times an hour outside dips. Dips in the saturation of $10 \%$ or more below the baseline value were studied. A stepped fall in saturation with steady pulse waveform and continued breathing movements suggested obstruction of the airways. These episodes were counted as incidents per hour and their length noted as
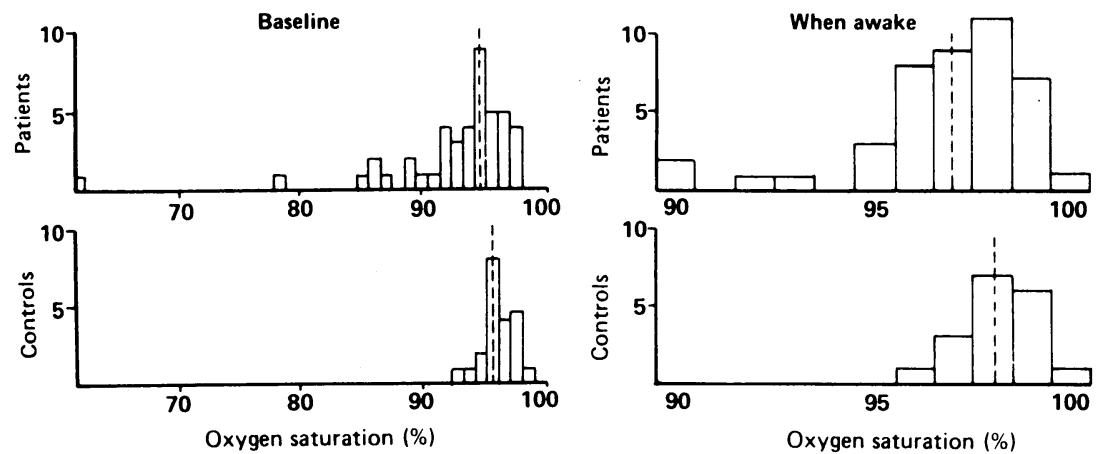

Results of monitoring oxygen saturation. Dashed lines show median values. Data are not normally distributed; for each measure the difference between patients and controls was highly significant (Mann-Whitney U test; $p \leqslant 0.005$ ) time of hypoxia per hour. Oxygen saturation when the child was awake was noted at the beginning or end of the night.

The figure shows the full results of measuring oxygen saturation. For all measures of hypoxaemia a substantial minority of the patients admitted for adenotonsillectomy fell completely outside the normal range. Fifteen children who suffered night time hypoxaemia were identified, with baseline oxygen saturation below $90 \%$ (nine patients) or at least one hypoxic episode an hour (13), or both (seven). Twelve children had episodes of hypoxia (totalling $\geqslant 1$ minute per hour). The 15 children who suffered night time hypoxia were then compared with the remaining 29 from the group admitted for adenotonsillectomy.

There were no notable differences between the groups regarding sex, history of snoring or apnoea, body weight, or any features found on examination of ears, palate, mandible, tonsils, or neck glands. The clinical features that did distinguish the hypoxic group were younger age (median $3.5 v 5.6$ years; $\mathrm{p}<0.01$ ), non-white ethnic origin $(80 \%(12 / 15) v 24 \%(7 / 29)$ $\mathrm{p}=0.001$ ), and physical signs of nasal obstruction namely, open mouth and audible respiration at rest $(93 \%(14 / 15) v 38 \%(11 / 29) ; \mathrm{p}=0.001)$. In addition, oxygen saturation when the patient was awake was lower (median $95 \%$ v 97\%; $<<0.001$ ).

Hypoxic patients could not be identified by clinical features alone, but by combining the presence or absence of breathing through the mouth with the oxygen saturation when awake we identified affected patients with $93 \%$ sensitivity $(95 \%$ confidence interval 70 to $100 \%$ ) and $86 \%$ specificity ( 73 to $99 \%$ ).

\section{Comment}

We showed that significant night time hypoxaemia is common, occurring in $34 \%$ of children admitted for adenotonsillectomy. We have clearly defined the importance of breathing through the mouth as a clinical sign of obstruction and the usefulness of measuring oxygen saturation when awake. Children who clearly breath through the mouth will suffer night time hypoxaemia unless their oxygen saturation when awake is at least $\mathbf{9 7 \%}$. We hope that this will help paediatricians and otorhinolaryngologists to assess children correctly and avoid unnecessary operations.

1 Brouillette RT, Fernbach SK, Hunt CE. Obstructive sleep apnea in infants and children. F Pediatr 1982;100:31-40.

2 Guilleminault C, Eldridge FL, Simmons FB, Dement WC. Sleep apnea in eigh children. Pediatrics 1976;58:23-31

3 Mauer KW, Staats BA, Olsen KD. Upper airway obstruction and disordered nocturnal breathing in children. Mayo Clin Proc 1983;58:349-53.

4 Grundfast KM, Wittich DJ. Adenotonsillar hypertrophy and upper airway obstruction in evolutionary perspective. Laryngoscope 1982;92:650-5.

5 Maw AR. Tonsillectomy today. Arch Dis Child 1986;61:421-3.

(Accepted 20 fanuary 1989) 